\title{
Excretory-secretory antigen is better than crude antigen for the serodiagnosis of clonorchiasis by ELISA
}

\author{
Min-Ho CHOI, Il Chan PARK, Shunyu LI and Sung-Tae HONG* \\ Department of Parasitology and Tropical Medicine, and Institute of Endemic Diseases, \\ Seoul National University College of Medicine, Seoul 110-799, Korea
}

\begin{abstract}
Although stool examination is the standard diagnostic method of clonorchiasis, serodiagnosis by ELISA using crude antigen is now widely used because of its convenience. However, ELISA diagnosis still suffers from cross-reactions, and therefore there is a need to improve the present conventional ELISA. The present study was undertaken to evaluate the diagnostic value of ELISA using excretory-secretory antigen (ESA) instead of crude antigen (CA) of Clonorchis sinensis. The diagnostic sensitivity of ELISA using excretory-secretory antigen was $92.5 \%$, which was higher than that of ELISA using crude Clonorchis sinensis antigen (88.2\%). In addition, the specificity of excretory-secretory antigen was found $93.1 \%$ while that of crude antigen was $87.8 \%$. In summary, Clonorchis sinensis ESA was found to be a better serodiagnostic antigen than CA for ELISA.
\end{abstract}

Key words: Clonorchis sinensis, ELISA, excretory-secretory antigen, crude antigen

\section{INTRODUCTION}

Clonorchis sinensis, the Chinese liver fluke, is a human parasitic helminth of the biliary tree, and is widely distributed in Korea, China, East Russia, Taiwan and Vietnam (Rim, 1986). The 6th nationwide survey on parasitic infections in Korea reported that the egg positive rate of $C$. sinensis was $1.4 \%$ over the country (Ministry of Health and Welfare, 1997). This egg positive rate represented the status of clonorchiasis among the general population, but the rate is considerably higher in endemic areas. Therefore about one million cases of clonorchiasis are estimated now in Korea.

Although stool examination is the standard

- Received 30 January 2003, accepted after revision 17 February 2003.

*Corresponding author (e-mail: hst@snu.ac.kr) diagnostic method for clonorchiasis, it is becoming difficult due to lack of compliance. In order to control clonorchiasis in endemic areas, effective mass screening is necessary; accordingly, serodiagnosis is now rapidly replacing the stool examination. Moreover, though the skin test is easy and rapid, it is not specific, and therefore, ELISA has been widely adopted (Lee et al., 1981; Hong, 1988; Chen et al., 1988; Yong et al., 2001).

Diagnosis by conventional ELISA, using the crude antigen (CA) of $C$. sinensis, is known to be sensitive and specific, but cross-reactions are known to occur (Hong, 1988; Sirisinha et al., 1990). In this context, more sensitive and specific sero-diagnosis tests are required for clonorchiasis.

The excretory-secretory antigen (ESA) of $C$. sinensis has been suggested to be a more sensitive and specific antigen than CA for diagnosis of active clonorchiasis (Kim, 1994, 1998). The present study 
was undertaken to evaluate the diagnostic value of ELISA using the ESA versus conventional ELISA, which uses the CA of $C$. sinensis.

\section{MATERIALS AND METHODS}

\section{Preparation of antigens}

Metacercariae of $C$. sinensis were collected from freshwater fish, Pseudorasbora parva, which were obtained from an endemic area in China. They were then fed orally to rabbits and the adult flukes were recovered from the livers 12 weeks later. The recovered flukes were chopped and homogenized in ice, centrifuged at $15,000 \mathrm{rpm}$ for 1 hour at $4^{\circ} \mathrm{C}$ and the supernatant obtained (CA) was stored at $-20^{\circ} \mathrm{C}$ until required. The protein concentration of the $\mathrm{CA}$ was determined to be $3.69 \mathrm{mg} / \mathrm{ml}$.

One hundred active moving worms which were recovered from rabbits were placed in $10 \mathrm{ml}$ of phosphate buffered saline (PBS) at $37^{\circ} \mathrm{C}$ for 15 hours. This PBS supernatant after centrifugation at 15,000 $\mathrm{rpm}$ at $4^{\circ} \mathrm{C}$ was used as ESA at a protein concentration of $0.67 \mathrm{mg} / \mathrm{ml}$.

\section{Collection of sera}

A total of 509 samples of human clonorchiasis sera were obtained at an endemic locality in Heilongjiang, China. All infected subjects had a life-long history of consuming raw freshwater fish and were egg positive by stool examination. Healthy control serum samples were obtained from 163 healthy students at the Seoul National University. Sera were separated and stored at $-70^{\circ} \mathrm{C}$. Sera obtained from patients with other trematode infections were also used for comparison purposed; Opisthorchis viverrini (47 cases), Schistosoma japonicum (14 cases), Paragonimus westermani (20 cases), and Fasciola hepatica (3 cases). The sera of $S$. japonicum infection were obtained from Jiangxi, China and O. viverrini from Tailand, all subjects passed eggs in feces. The sera of $P$. westermani and $F$. hepatica infection were obtained from patients who were confirmed either parasitologically or pathologically.

\section{ELISA}

ELISA was performed in polystyrene EIA microtitration 96-well flat-bottomed plates (Nunc
Immunoplate I). Carbonate-bicarbonate buffer ( $\mathrm{pH}$ 9.6) and phosphate buffered saline $(\mathrm{pH}$ 7.2) containing Tween 20 (PBST) were used for the coating procedures. For plate coating, $0.1 \mathrm{ml}$ of antigen solution, protein concentration of which was $2 \mu \mathrm{g} / \mathrm{ml}$, was added to each well. The plates were then sealed and incubated at $4^{\circ} \mathrm{C}$ overnight. Blocking solution ( $1 \%$ bovine saline serum albumin in PBS, 0.5 $\% \mathrm{NaN}_{3}$ ) was added after washing three times with PBST and incubating overnight. Subsequently, diluted (1:25) of test sera were added to the wells, and the plates were then incubated at $37^{\circ} \mathrm{C}$ for one hour and washed with PBST. After shaking dry, 0.1 $\mathrm{ml}$ of diluted $(1: 50,000)$ horseradish peroxidaselabeled sheep IgG against human immunoglobulins (US Biochemical Cooperation) was added and incubated again for one hour at $37^{\circ} \mathrm{C}$. The plates were then washed 3 times with PBST, and freshly prepared substrate solution (tetramethylbenzidine and $0.1 \% \mathrm{H}_{2} \mathrm{O}_{2}$ ) was then added to each well. Following incubation at $37^{\circ} \mathrm{C}$ for half an hour, the optical density was read at $450 \mathrm{~nm}$ using a Titertek Multiskan (Flow Laboratories). Samples with an absorbance of $<0.25$ were deemed negative and those $\geq 0.25$ as positive.

\section{RESULTS}

\section{Absorbances by ELISA using CA and ESA}

Fig. 1 and Table 1 show the distribution and the mean absorbances of the normal controls, and of the

Table 1. Comparison of mean absorbances with crude antigen and excretory-secretory (ES) antigen of Clonorchis sinensis

\begin{tabular}{lrcc}
\hline \multirow{2}{*}{$\begin{array}{c}\text { Infected serum } \\
\text { of }\end{array}$} & No. & \multicolumn{2}{c}{ Absorbances (Mean \pm S.D.) } \\
\cline { 3 - 4 } & & Crude antigen & ES antigen \\
\hline Control & 163 & $0.066 \pm 0.058$ & $\left.0.074 \pm 0.039^{\mathrm{a}}\right)$ \\
C. sinensis & 509 & $0.353 \pm 0.115$ & $\left.0.404 \pm 0.129^{\mathrm{b}}\right)$ \\
O. viverrini & 47 & $0.261 \pm 0.135$ & $0.212 \pm 0.125^{\mathrm{c}}$ \\
S. japonicum & 14 & $0.093 \pm 0.124$ & $\left.0.079 \pm 0.078^{\mathrm{d}}\right)$ \\
P. westermani & 20 & $0.188 \pm 0.071$ & $\left.0.144 \pm 0.057^{\mathrm{e}}\right)$ \\
F. hepatica & 3 & $0.123 \pm 0.059$ & $\left.0.104 \pm 0.057^{\mathrm{f}}\right)$ \\
\hline
\end{tabular}

The differences were statistically analyzed by Student $t$-test using SPSS 10.0 for Windows program.

a) $\mathrm{p}=0.135(\mathrm{p}>0.05),{ }^{b} \mathrm{p}=0.001(\mathrm{p}<0.01)$,

c) $p=0.075(p>0.05),{ }^{d)} p=0.707(p>0.05)$,

e) $p=0.038(p<0.05),{ }^{\text {f) }} \mathrm{p}=0.711(\mathrm{p}>0.05)$. 


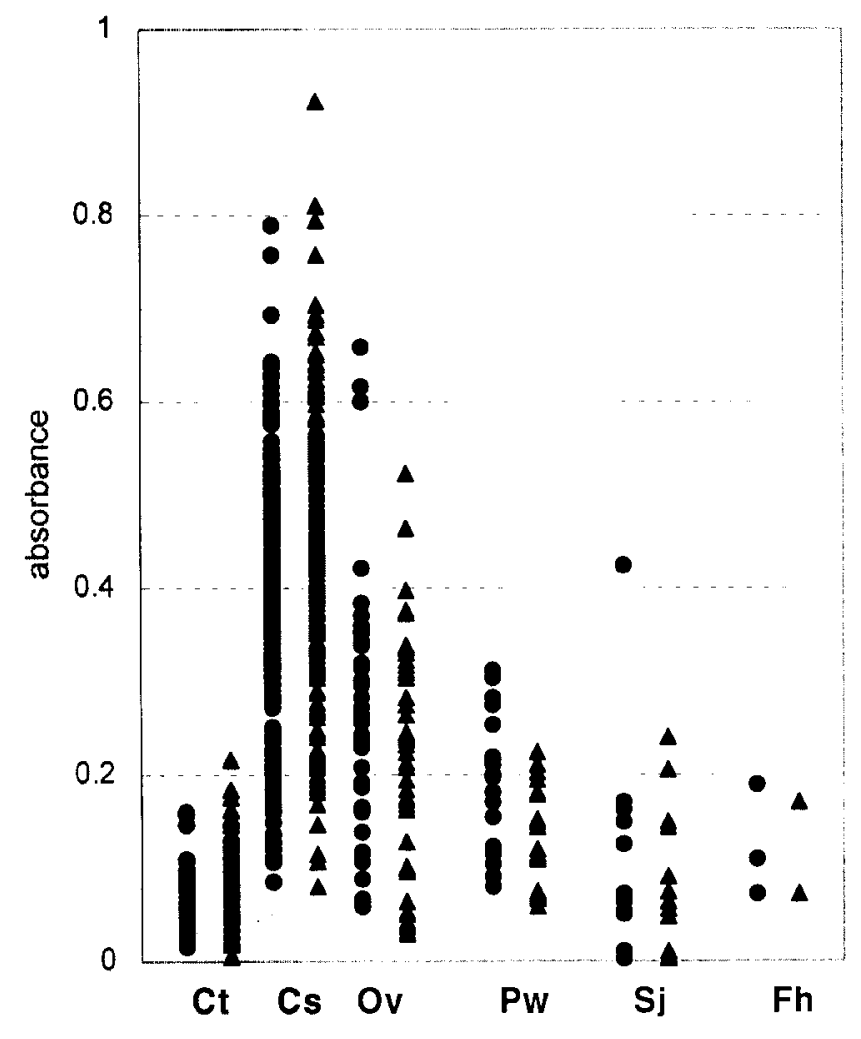

Fig. 1. Distribution of absorbances of various tested sera with crude antigen $(\bullet)$ and excretory-secretory antigen $(\boldsymbol{A})$ of Clonorchis sinensis. Ct, Control; Cs, Clonorchis sinensis; Ov, Opisthorchis viverrini; Pw, Paragonimus westermani; $\mathrm{Sj}$, Schistosoma japonicum; Fh, Fasciola hepatica.

Table 2. Comparison of sensitivity between crude antigen and excretory-secretory antigen of Clonorchis sinensis

\begin{tabular}{lccc}
\hline & $\begin{array}{c}\text { No. of } \\
\text { sera }\end{array}$ & $\begin{array}{c}\text { No of } \\
\text { positive }\end{array}$ & $\begin{array}{c}\text { Sensitivity } \\
(\%)\end{array}$ \\
\hline Crude antigen & 509 & 449 & 88.2 \\
Excretory-secretory antigen & 509 & 471 & $92.5^{\mathrm{a})}$ \\
\hline
\end{tabular}

Pearson's chi-square test was applied using SPSS 10.0 for Windows.

a) $\mathrm{p}=0.019(\mathrm{p}<0.05)$. infected individuals. Mean ELISA absorbance for the CA of the serum samples of the controls was very low and individual absorbances showed little variation. Absorbances of the sera of clonorchiasis for CA much increased to mean level of $0.353 \pm 0.058$ (Table 1). Mean absorbances of the sera of other trematodiases for CA remained a little higher than 0.1 .

The absorbances of serum samples of the controls for ESA were in quite similar level with those for CA. Mean absorbances of serum samples of clonorchiasis for ESA increased to $0.404 \pm 0.129$ and those of other trematodiases also increased a little (Table 1).

Sensitivity and specificity: Using the negativity/ positivity criterion described above, the sensitivity of ESA-ELISA was $92.5 \%$, which was higher than that of CA-ELISA, $88.2 \%$ (Table $2, \mathrm{P}<0.05, \mathrm{x}^{2}=5.52$ ). Sera of the controls and those with F. hepatica infection showed no cross-reactions either as CA or ESA, but the sera of those infected with $O$. viverrini, $S$. japonicum, and $P$. westermani cross-reacted with both CA and ESA (Table 3). The results of our study show that ESA was more specific than CA as $93.1 \%$ versus $87.8 \%$.

\section{DISCUSSION}

Clonorchis sinensis ESA proved to be a better serodiagnostic antigen than CA for ELISA. The diagnostic sensitivity of ELISA with ESA was 92.5\% while that of ELISA with CA was $88.2 \%$, when 509 human sera of clonorchiasis were screened in the present study, which was statistically significant. The diagnostic specificity of ELISA with ESA was increased to $93.1 \%$ from $87.8 \%$ by using CA.

Table 3. Comparision of specificity between crude antigen and excretory-secretory (ES) antigen of Clonorchis sinensis

\begin{tabular}{|c|c|c|c|c|c|}
\hline & \multirow{2}{*}{$\begin{array}{c}\text { No. of } \\
\text { samples }\end{array}$} & \multicolumn{2}{|c|}{ Crude antigen } & \multicolumn{2}{|c|}{ ES antigen } \\
\hline & & No. negative & Specificity (\%) & No. negative & Specificity (\%) \\
\hline Control & 163 & 163 & 100 & 163 & 100 \\
\hline O. viverrini & 47 & 23 & 48.9 & 30 & $63.8^{\mathrm{a})}$ \\
\hline S. japonicum & 14 & 13 & 92.9 & 14 & $100^{b)}$ \\
\hline P. westermani & 20 & 15 & 75.0 & 20 & $100^{c)}$ \\
\hline F. hepatica & 3 & 3 & 100 & 3 & 100 \\
\hline Total & 247 & 217 & 87.8 & 230 & $93.1^{\mathrm{d})}$ \\
\hline
\end{tabular}

Pearson's chi-square test was applied using SPSS 10.0 for Windows.

$\left.{ }^{a)} \mathrm{p}=0.140(\mathrm{p}>0.05),{ }^{b} \mathrm{p}=0.309(\mathrm{p}>0.05),{ }^{c}\right) \mathrm{p}=0.017(\mathrm{p}<0.05),{ }^{\mathrm{d}} \mathrm{p}=0.037(\mathrm{p}<0.05)$. 
Especially cross-reactivity of ESA with serum specimens of opisthorchiasis, schistosomiasis and paragonimiasis was reduced compared to that of CA.

C. sinensis ESA was found to include proteins of 7$8,12.5$, and $30 \mathrm{kDa}(\mathrm{Kim}, 1998)$, and the 7 or $8 \mathrm{kDa}$ protein predominated (Chung et al., 2002). ESA has been suggested to be a valuable candidate diagnostic antigen of active clonorchiasis in rabbits and humans (Kim, 1994 \& 1998). The protein bands in C. sinensis ESA were also found to form 7-8, 17 and $26-28 \mathrm{kDa}$ bands, while in CA bands at $35,43,55$, and $70 \mathrm{kDa}$ were additionally observed (Hong et al., 1997, 1999).

The 7-8 $\mathrm{kDa}$ band was found to be a mixture of two proteins of 7 and $8 \mathrm{kDa}$. These were found to be concentrated mainly in the tegument and the seminal receptacle by immunohistochemistry (Chung et al., 2002). The protein band possibly resulted from the partition of a larger complexed protein. Moreover the purification of the 7 and $8 \mathrm{kDa}$ band would further improve diagnostic efficacy.

The other major components in C. sinensis ESA were at 12.5 and $17 \mathrm{kDa}$. The $17 \mathrm{kDa}$ band has been previously identified as a cysteine proteinase (Chung et al., 2000). This band is not strongly antigenic but its polyclonal antibody was found to cross-react with a similarly sized band in other trematode species. Therefore, if this band were excluded from the ESA, its specificity should be improved. The characteristics of the band at $12.5 \mathrm{kDa}$ are unknown (Kim, 1998).

The third component of $C$. sinensis ESA was bands at 26 and $28 \mathrm{kDa}$. These represent a single protein type, which was previously identified as the enzyme, glutathione S-transferase. The $28 \mathrm{kDa}$ protein was found to be composed of 212 amino acids (Kang et al., 2001). Moreover, these two bands were found to be antigenic and showed low level of cross-reactivity and are therefore, regarded as a potential diagnostic antigen (Choi et al., 2003). The protein band recorded at $30 \mathrm{kDa}$ (Kim, 1998) may possibly be same with the $28 \mathrm{kDa}$ band (Kang et al., 2001), mainly due to different system of size estimation.

The $C$. sinensis ESA of the present study was observed to increase the diagnostic sensitivity and specificity by ELISA. This increased sensitivity may be due to the higher levels the 7-8 and 26-28 $\mathrm{kDa}$ bands in ESA. On the other hand, CA contains several other protein bands and thus the major antigenic proteins are both much diluted and represent only a smaller part of the soluble protein present. Of the several additional proteins in the crude antigen of $C$. sinensis, those at 35 and $70 \mathrm{kDa}$ have been found to be cross-reactive with the sera of other helminthiases (Hong et al., 1999). Conversely ESA contains higher levels of the 7-8, 17, and 26-28 $\mathrm{kDa}$ proteins and little 35 or $70 \mathrm{kDa}$ protein, which explains the observed increase in diagnostic specificity.

It is quite reasonable to speculate that most of the present subjects of clonorchiasis were cases of rather chronic than acute infection because they lived in a infection status of natural equilibrium. In acute infection, serological reaction is known to be first induced to the glycoprotein which is rich in the ESA. This phenomenon may influence the difference in the sensitivity between ESA and CA if the subjected cases were in acute stage of infection. In the present study, however, the influence by the infection stage is negligible because most of the subjects were in the stage of chronic infection.

ESA has also been reported to be a good antigen for the serodiagnosis of paragonimiasis. The ESA of Paragonimus westermani eggs was also found to be a better test substrate than CA (Cho et al., 2000). The proteins in the ESA of $P$. ohirai were also found to be allergenic and thus useful for serodiagnosis (Ikeda, 2002).

ELISA is now routinely used in Korea for the diagnosis of several helminthiases, which include clonorchiasis. The ELISA diagnosis may be improved if ESA were applied instead of CA. However, the difficulty of obtaining sufficient ESA presents a practical limitation. Further study on the proteinaceous antigenic components in ESA may enable the future production of recombinant antigen proteins.

\section{ACKNOWLEDGMENTS}

The authors appreciate kind help of Prof. SeungYull Cho, Department of Molecular Parasitology, Sungkyunkwan University College of Medicine for donating paragonimiasis and fascioliasis sera. We also thank Dr. Honggen Chen, Jiangxi CDC, China for donating sera of schistosomiasis, and the staff of 
Heilongjiang CDC, China for helping in the collection of clonorchiasis sera, and finally Ms. SungHyun Kum for performing the ELISA.

\section{REFERENCES}

Chen CY, Hsieh WC, Shih HH, Chen SN (1988) Immunodiagnosis of clonorchiasis by enzyme-linked immunosorbent assay. Southeast Asian J Trop Med Public Health 19: 117-121.

Cho SY, Kong Y, Yun DH, et al. (2000) Persisting antibody reaction in paragonimiasis after praziquantel treatment is elicited mainly by egg antigens. Korean J Parasitol 38: 7584.

Chung YB, Chung BS, Choi MH, Chai JY, Hong ST (2000) Partial characterization of a $17 \mathrm{kDa}$ protein of Clonorchis sinensis. Korean J Parasitol 38: 95-97.

Chung YB, Lee M, Yang HJ, et al. (2002) Characterization of partially purified $8 \mathrm{kDa}$ antigenic protein of Clonorchis sinensis. Korean J Parasitol 40: 83-88.

Hong ST (1988) Changes of anti-Clonorchis sinensis IgG antibody in serum after praziquantel treatment in human clonorchiasis. Korean J Parasitol 16: 1-8.

Hong ST, Kho WG, Lee M, Lee JS, Lee SH (1997) Immunoblot patterns of clonorchiasis. Korean J Parasitol 35: 87-93.

Hong ST, Lee M, Sung NJ, Cho SR, Chai JY, Lee SH (1999) Usefulness of IgG4 subclass antibodies for diagnosis of human clonorchiasis. Korean J Parasitol 37: 243-248.

Ikeda T (2002) Identification and characterization of major allergens in excretory-secretory products of the worm Paragonimus ohirai. Int Arch Allergy Immunol 128: 187-194.

Kang SY, Ahn IY, Park CY, et al. (2001) Clonorchis sinensis: Molecular cloning and characterization of 28-kDa glutathione S-transferase. Exp Parasitol 97: 186-195.

Kim SI (1994) Immune reactions between excretory-secretory antigens and specific antibodies of Clonorchis sinensis before and after praziquantel treatment in experimentally infected rabbits. Korean J Parasitol 32: 35-42.

Kim SI (1998) A Clonorchis sinensis-specific antigen that detects active human clonorchiasis. Korean J Parasitol 36: 37-45.

Lee JK, Min DY, Im KI, Lee KT, Soh CT (1981) Study of enzyme-linked immunosorbent assay (ELISA) method in serodiagnosis of Clonorchis sinensis infection. Yonsei J Med 14: 133-147.

Ministry of Health and Welfare and Korea Association of Health (1997) The prevalence of intestinal parasitic infection in Korea-The sixth report. Seoul.

Rim HJ (1986) The current pathobiology and chemotherapy of clonorchiasis. Korean J Parasitol 24(suppl): 5-141.

Sirisinha S, Sahassananda D, Bunnag D, Rim HJ (1990) Immunological analysis of Opisthorchis and Clonorchis antigens. J Helminthol 64: 133-138.

Yong TS, Yang HJ, Park SJ, Kim YK, Lee DH, Lee SM (1998) Serodiagnosis of clonorchiasis using a recombinant antigen. Korean J Parasitol 36: 183-190. 